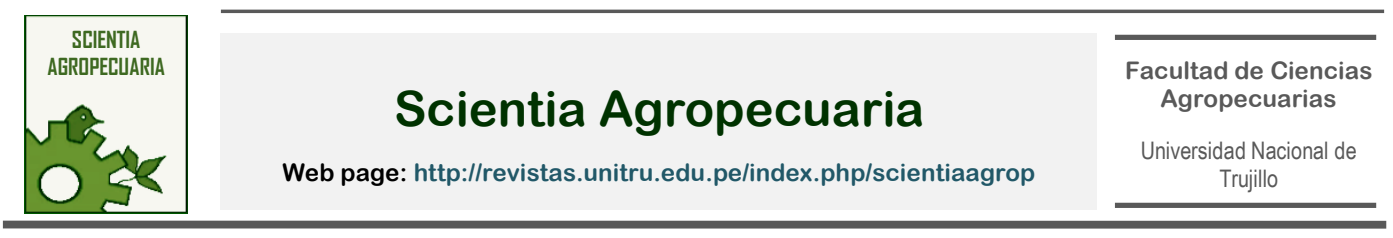

\title{
REVIEW
}

\section{The murta (Ugni molinae) and its beneficial health properties: A review}

\author{
La murta (Ugni molinae) y sus propiedades benéficas para la salud: Una revisión

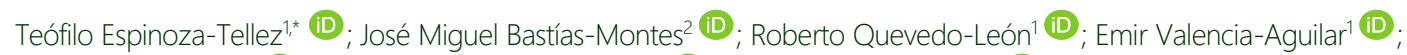 \\ Oscar Díaz-Carrasco $\mathbb{D}^{\mathbb{D}}$; Miguel Ángel Solano-Cornejo3 ${ }^{\mathbb{D}}$; Francisco Mesa-Mesina $4 \mathbb{D}$
}
Departamento de Acuicultura y Recursos Agroalimentarios, Programa Fitogen, Universidad de Los Lagos, Av. Alberto Fuchslocher 1305, Región de los Lagos, Osorno. Chile.
2 Escuela Ingeniería en Alimentos, Universidad del Bío-Bío, Av. Andres Bello 720, Chillán. Chile.
3 Escuela de Ingeniería de industrias Alimentarias, Universidad Nacional Pedro Ruiz Gallo, Avenida Juan XXIII No 391 Lambayeque. Peru.
4 Departamento de Ciencias Administrativas y Económicas, Universidad de Los Lagos, Av. Alberto Fuchslocher 1305, Región de los Lagos, Osorno. Chile.

*Corresponding author: teofilo.espinoza@ulagos.cl (T. Espinoza-Tellez).

Received: 21 July 2020. Accepted: 22 January 2021. Published: 10 March 2021.

\begin{abstract}
Currently there is a great demand for food products containing functional properties and/or bioactive compounds. One of the fruits that has been widely studied is the murta (Ugni molinae), which appears to contain various beneficial health properties. This review compiles scientific information on the functional and beneficial properties of murta for human health, such as the main components associated with anti-inflammatory activity, antimicrobial activity, antioxidant activity, nutritional composition, other uses of murta, economic aspects, and current and future challenges of murta, all based on updated scientific work.
\end{abstract}

Keywords: murta; flavonoids; antioxidants; antibacterial; anti-inflammatory; functional food.

\begin{abstract}
Resumen
Actualmente existe una gran demanda de productos alimenticios que contengan propiedades funcionales y/o compuestos bioactivos. Uno de los frutos que ha sido ampliamente estudiado es la murta (Ugni molinae), el cual parece contener diversas propiedades benéficas para la salud. En la presente revisión se recopila información científica de las propiedades funcionales y benéficas de la murta para la salud humana; como, por ejemplo, los principales componentes asociados a la actividad antiinflamatoria, actividad antimicrobiana, actividad antioxidante, composición nutricional, otros usos de la murta, aspectos económicos, retos actuales y futuros de la murta; todo basado en trabajos científicos actualizados.
\end{abstract}

Palabras clave: murta; flavonoides; antioxidantes; antibacteriana; antiinflamatorio; alimento funcional.

DOI: https://dx.doi.org/10.17268/sci.agropecu.2021.14

Cite this article:

Espinoza-Tellez, T., Bastías, J., Quevedo-León, R., Valencia-Aguilar, E., Díaz-Carrasco, O., Solano, A. A., \& Mesa-Mesina, F. (2021). La murta (Ugni molinae) y sus propiedades benéficas para la salud: Una revisión. Scientia Agropecuaria, 12(1), 121-131.

\section{Introducción}

Los beneficios de los berries están dados principalmente por la presencia de compuestos fenólicos, como por ejemplo las antocianinas, las que han sido ampliamente estudiadas por sus propiedades bioactivas que incluyen propiedades antioxidantes, anticancerígenas y antiinflamatorias (Chile \& Adimark, 2014). El desarrollo de alimentos y productos alimenticios que mejoren la salud y el bienestar se ha convertido en un foco de investigación principal en la industria alimentaria, consiguiéndose productos alimenticios enriquecidos con compuestos funcionales como pre y probióticos, vitaminas, minerales, fibra dietética, aceites omega 3 y esteroles vegetales, entre otros (Zura-Bravo et al., 2019; Zura-Bravo, Rodriguez, Stucken, \& Vega-Gálvez, 2018). Un fruto a destacar es la murta, berries que presenta un alto valor nutricional (Fuentes, Figueroa, Valdenegro, \& Vinet, 2019; Santander, Osorio, \& Mejia- España, 2017; SchmedaHirschmann, Jiménez-Aspee, Theoduloz, \& Ladio, 2019) y se caracteriza por su contenido de componentes beneficos para la salud (Kamiloglu et al., 2016; López de Dicastillo et al., 2017; J. López, Vega-Gálvez, Rodríguez, 
Uribe, \& Bilbao-Sainz, 2018; Rubilar et al., 2011). El constante desarrollo científico de la química en los últimos años ha permitido ir mejorando el conocimiento de sus principios activos (D'Urso, Montoro, Lai, Piacente, \& Sarais, 2019; Indap, 2018; Lorca, 2018; Rodriguez et al., 2014; Schmeda-Hirschmann et al., 2019). De este punto de vista se puede considerar a la murta como una planta medicinal, que presenta un alto potencial económico dentro de las bayas nativas, siendo un fruto de fácil obtención, especialmente en el sur de Chile. Los berries han sido catalogados como frutos con importantes propiedades beneficiosas para la salud humana (Avello, Pastene, Bustos, Bittner, \& Becerra, 2013; Riquelme et al., 2014), los cuales son asociados a la defensa de diferentes patologías y aplicaciones en el tratamiento y prevención del cáncer. por ejemplo, se ha demostrado que los componentes hidrosolubles de hojas de Ugni molinae tienen efecto sobre la viabilidad de células de cáncer gástrico en humanos (ArancibiaRadich et al., 2016; Avello, Pastene, \& Eillen, 2020; Goity et al., 2013); así como las actividades biológicas, antimicrobianas y antioxidantes (López de Dicastillo et al., 2017).

En los extractos de semilla de murta se encuentran presentes metabolitos secundarios tales como ácidos fenólicos, flavonoides y taninos, considerados como precursores de defensa contra el ataque de patógenos relacionadas con la salud humana y alimentaria (Cabrera-Barjas et al., 2020).

Se han evaluado también bayas de murta deshidratadas enriquecidas con bacterias probióticas, (Lactobacillus casei var. Rhamnosus). Algunos tratamientos han incluido tecnología de vacío y secado por convección (Alfaro, Mutis, Quiroz, Seguel, \& Scheuermann, 2014; Pirce et al., 2020; Scheuermann et al., 2014), para producir bayas de murta enriquecidas con probióticos (Zura-Bravo et al., 2019; Zura-Bravo et al., 2018). El objetivo de la presente revisión es mostrar investigaciones de los últimos 5 años sobre los beneficios de la murta en la salud humana, en cuanto a sus principales componentes asociados a la actividad antiinflamatoria, actividad antimicrobiana, actividad antioxidante, composición nutricional y otros usos de la murta, aspectos económicos, además de retos actuales y futuros.

\section{Características morfológica \\ 2.1 Descripción botánica}

La murta (Ugni molinae) familia de las myrthaceae (Lorca, 2018; Retamales, Scherson, \& Scharaschkin, 2014), también conocida como murtilla, mirto, murillo, magnoliopsida división magnoliophyta es una especie arbustiva endémica de Chile (Alarcón et al., 2019; Rodríguez et al., 2015). Es un arbusto de 1,8 $\mathrm{m}$ de altura de gran follaje que crece en el sur de Chile; de hojas pecioladas aovadooblongas con ápice agudo de 2,0 a 2,5 centímetros de longitud. Los frutos presentan una alta variabilidad en cuanto a color (color rosado o amarillo claro y rojo intenso), tamaño $(0,7$ a $1,3 \mathrm{~cm}$ de diámetro) y peso $(0,25$ a 0,40 g), de sabor dulce, aromático (Seguel \& Torralbo, 2004; Ulloa-Inostroza et al., 2017). La lámina de las hojas de U. molinae es discolora, sub-coriácea a coriácea, ovada a elíptico-lanceolada, apiculada, de borde entero, márgenes algo revolutos y base cuneada a obtusa. La lámina mide hasta $50 \mathrm{~mm}$ y el pecíolo hasta $7 \mathrm{~mm}$ de longitud. Una yema apical de U. molinae consta de una secuencia linear de primordios de entrenudos, nudos y nomofilos; estas yemas no presentan hojas modificadas (catafilos) y forman la cubierta externa. Cada fruto contiene entre 4 a 29 semillas y el largo de la semilla varía entre 1 a 2 mm, el peso de la semilla esta entre 0,1 a 21 $\mathrm{mg}$ con un peso promedio por semilla de $0,85 \mathrm{mg}$ (Atala \& Moya-Urrutia, 2014; Guenuleo et al., 2020). En la Figura 1 se muestran las características morfológicas de la murta.

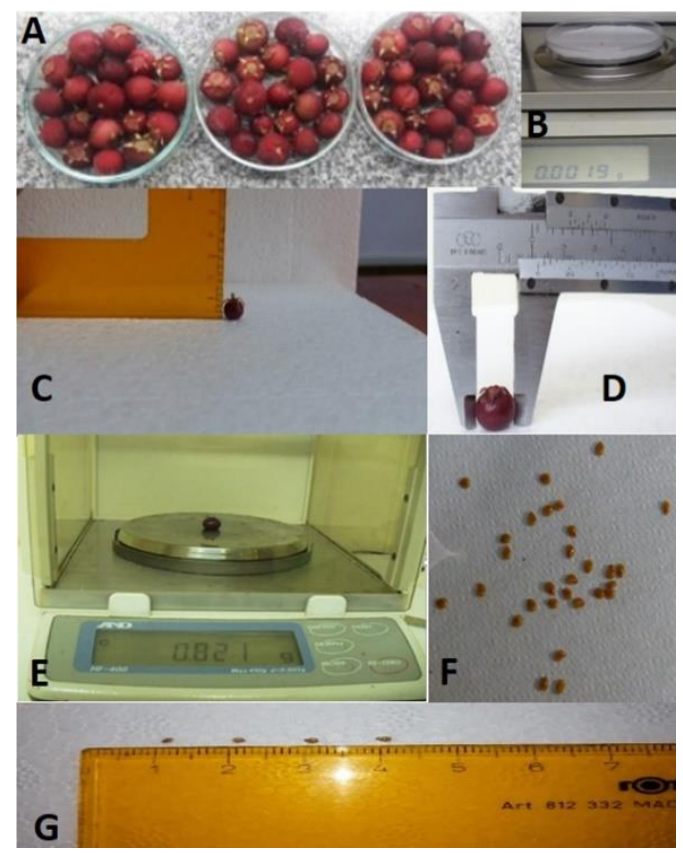

Figura 1. Características morfológicas de la murta: A) Murta recolectada en la comuna de San Juan de la Costa, Osorno, Chile. B) Peso de la semilla. C) Altura del fruto. D) Diámetro del fruto. E) Peso del fruto. F) Semillas (29) encontradas en un fruto de murta. G) Diámetro de la semilla de murta.

\subsection{Distribución geográfica}

La murta es un arbusto conocido desde antes de la época colonial y se encuentra de manera silvestre en la precordillera y cordillera de los Andes, en algunas zonas del centro sur comprendida entre la VII y la X Región de Chile y Argentina. Esta planta crece en terrenos de pobre nutrición, poco aptos para el cultivo de otras especies; sin embargo, también se ha domesticado e introducido en el Reino Unido y Australia con fines ornamentales (Lorca, 2018; Peña-Cerda et al., 2017; Rodríguez et al., 2016). A partir de prospecciones y recolecciones de germoplasma realizadas en 1996 por INIA Carillanca (Chile) se observó que la mayor frecuencia de la especie se presenta desde la depresión intermedia $\left(72^{\circ} \mathrm{LE}\right)$ hacia la Cordillera de la Costa ( $74^{\circ}$ LE) y desde los 10 hasta 300 msnm. En Chile el clima que caracteriza a los sectores en donde se desarrolla la murta en la VII Región es del tipo mediterráneo marino, en tanto, en la IX y X Región, las condiciones climáticas son más variables, pudiéndose encontrar en zonas de clima del tipo marino húmedo patagónico, marino cálido, 
marino frío y marino fresco. El período de recolección de frutos depende de la zona agroecológica, esta faena se inicia en la VII Región a mediados en febrero y finaliza a mediados de marzo. En cambio, en la IX Región, la recolección de fruto comienza en marzo y finaliza a fines de abril, y para la X Región, especialmente en Chiloé, la mayor cantidad de fruta para recolección se obtiene desde abril a mayo (Aguila \& Nahuelhual, 2008).

\section{Propiedades químicas}

La murta contiene heteropolisacáridos entre ellos las sustancias pécticas (Chakraborty et al., 2019) y la fibra dietética, la cual está compuesta principalmente de lignina, celulosa, pectina y hemicelulosa. Además, contiene ácidos grasos poliinsaturados (89,0\%), ácidos grasos monoinsaturados $(7,7 \%)$ y ácidos grasos saturados (3,3\%). Estos además se encuentran principalmente dentro de la semilla. Las pectinas están formadas por unidades de ácido galacturónico y se ubican en la pared celular de plantas terrestres, proporcionando consistencia y resistencia mecánica a los tejidos vegetales (Taboada et al., 2010). La presencia de compuestos nutricionales en semillas de murta como, fibra dietética, minerales y proteínas, puede transformarse en una fuente valiosa de fibra y compuestos polifenólicos; que son una alternativa viable para el desarrollo de alimentos funcionales (Cabrera-Barjas et al., 2020). La murta contiene polifenoles en sus hojas y frutos que podrían retardar la peroxidación lipídica y limitar el daño de los radicales libres (Alfaro et al., 2014; Lorca, 2018; Peña-Cerda et al., 2017; Ulloa-Inostroza et al., 2017). También se han reportado que las semillas de murta contienen: fibra cruda (64\%), grasa bruta (14\%), proteína bruta (12\%) de cenizas $(1,5 \%)$ y minerales $(0,04-0,23 \%)$ (Cabrera-Barjas et al., 2020). La semilla es una fuente potencial de aceite comestible, y puede constituir un suplemento dietético de buena calidad (Lorca, 2018; Rodríguez et al., 2016).

En las hojas de la murta se han encontrado taninos del tipo elagitanino y galotanino, flavonoides tales como la miricetina y la quercetina en forma de geninas y glucósidos; además, se ha evidenciado la presencia de flavan-3-oles como catequinas y ácidos fenólicos, con contenido de ácido linoleico (Alfaro et al., 2014; ArancibiaAvila et al., 2011; López et al., 2017; Lorca, 2018; PeñaCerda et al., 2017) y otros componentes como el ácido ursólico, ácido alfitólico y ácido corosólico. En extractos de hoja con acetato de etilo, se han encontrado cumarinas, taninos y ácidos fenólicos, así como ácido gálico, catequina, ácido cafeico, además de epicatechin. También se han obtenidos extractos con etanol, conteniendo fenoles totales $(49,5 \% \mathrm{v} / \mathrm{v})$, lo que produjo 18,39 y 26,14 mg AGE g ${ }^{-1}$ materia seca (Augusto-Obara, Pirceb, Scheuermannb, Spoto, \& Vieira, 2017). Suwalsky and Avello (2014) reportaron 10,64 mM equivalentes de ácido gálico en extractos de fruta. En extracto con metanol, se han encontrado polifenóles como: cumarinas, taninos, ácidos fenólicos, ácido gálico y catequina (Ma et al., 2019); así como heterósidos de los flavonoides como: miricetina, canferol, quercetina y otros triterpenoides, como el ácido alfitólico, asiático, betulínico, corosólico, oleanólico, ursólico, madecásico (Cabrera-Barjas et al., 2020; Goity et al., 2013).
Las antocianinas han sido estudiadas y probadas como agentes quimiopreventivos (Ma et al., 2019), así como de tener un amplio espectro de funciones biomédicas que ayudan a mejorar los trastornos cardiovasculares y el avance progresivo del estrés oxidativo inducido por la edad; así como las respuestas inflamatorias y diversas enfermedades degenerativas. Las antocianinas de los berries también mejoran las funciones neuronales y cognitivas del cerebro, la salud ocular y protegen el ADN genotipo. Los berries en general han tenido un impacto efectivo en diversas enfermedades crónicas, como la obesidad, el cáncer, las enfermedades cardiovasculares y neurodegenerativas (Ramirez, Zambrano, Sepúlveda, Kennelly, \& Simirgiotis, 2015).

Sin embargo, existen ciertos riesgos de consumir berries debido a la presencia de metales pesados; por ejemplo, es sabido que los berries cosechados a orillas de caminos por donde transitan vehículos a combustión; tienen altos contenidos de metales pesados debido a los gases de combustión. Aunque los minerales constituyen una parte importante de la dieta, estos pueden acelerar la formación de componentes tóxicos en la fruta al contacto con el ambiente en zonas urbanas; por ejemplo, al contacto con fertilizantes con el smog, con la lluvia, con desechos municipales e industriales y con aguas contaminadas para regadíos. Es sabido que las plantas absorben una gran cantidad de elementos que pueden incorporarse a la fruta, lo que plantea riesgos para la salud humana (Nowakowska, Ochmian, \& Mijowska, 2017; Pereira et al., 2018; Petousi et al., 2019).

\section{Composición nutricional}

Los berries pueden considerarse una importante fuente de vitaminas, minerales y fibras con componentes funcionales (López et al., 2018), por ejemplo, la murta tiene una composición rica y diversificada de bioactivos, compuestos con propiedades nutricionales para la salud (Augusto-Obara et al., 2017; Indap, 2018; Rodriguez et al., 2014). Diversos estudios han demostrado que, en el procesamiento de la murta, por ejemplo, en un proceso de secado la transferencia de calor y de humedad afecta a los nutrientes, dando lugar a cambios físicos como el colapso de la pared celular y la contracción de la textura; cambiándole las características sensoriales colorantes, textura, sabor y nutricionales, antioxidantes, vitaminas, polifenoles (López, Bustos, Guarda, \& Galotto, 2016; López de Dicastillo et al., 2017; Lopez et al., 2016). Conocer el perfil y la abundancia de los compuestos nutricionales en los alimentos, es de suma importancia para evaluar los beneficios para la salud que estos confieren. La biodisponibilidad depende del tipo y concentración de cada compuesto bioactivo. En el caso de los compuestos fenólicos estos llegan rápidamente al intestino grueso (Parkar, Stevenson, \& Skinner, 2008), donde pueden ser metabolizados por la microbiota intestinal. Algunas bacterias tales como los lactobacilos forman parte de la microbiota intestinal, y sintetizan componentes activos como: $\beta$-glucosidasas y enzimas descarboxilasas del ácido p-cumárico (Jaiswal \& Abu-Ghannam, 2013; Zura-Bravo et al., 2019; Zura-Bravo et al., 2018). En la Tabla 1 se reporta la composición química y nutricional en la murta. 
Tabla 1

Composicion química y nutricional en la murta (Ugni molinae)

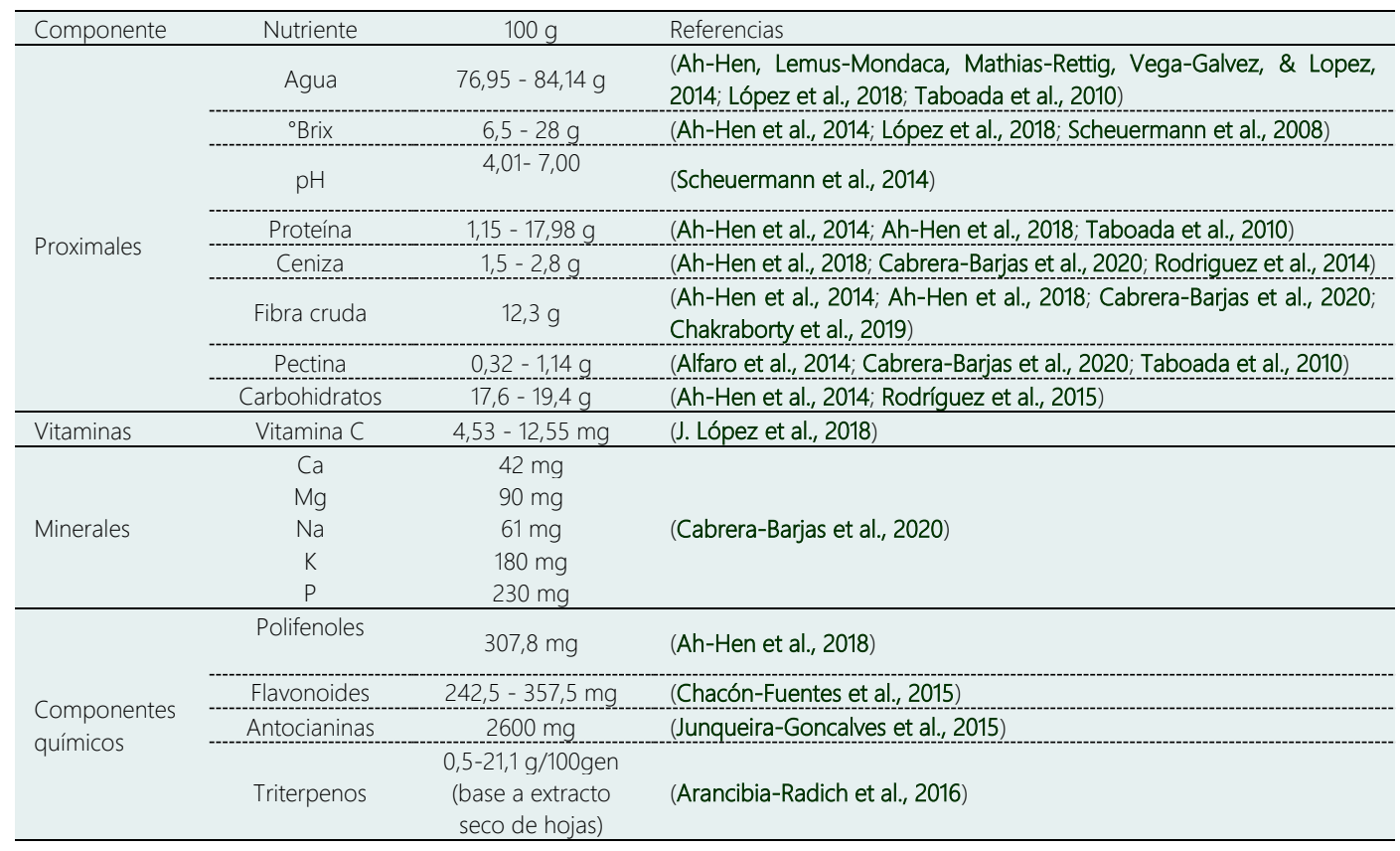

\section{Propiedades funcionales}

La murta es un vegetal endémico, perteneciente a la familia de las Myrtáceae, productora de bayas cuyo aroma característico ha sido descrito como una mezcla de notas frutales, dulces y florales debido a sus compuestos volátiles, similares a los encontrados en muchos frutos aromáticos tropicales (Scheuermann et al., 2014; Scheuermann et al., 2008). En la zonas sur de Chile, los frutos de esta especie son altamente consumidos como fruto fresco (Lorca, 2018), siendo también procesados como mermelada, conservas en almíbar, utilizados en repostería o en la elaboración de jugos y se ha demostrado que estos frutos poseen una rica y diversa composición de compuestos bioactivos (López et al., 2017; Radrigán, Aqueveque, \& Ocampo, 2017) con propiedades medicinales (Ah-Hen et al., 2018), los cuales han sido intensamente estudiados por los aportes a la salud de sus propiedades funcionales (Lemus-Mondaca, Ah-Hen, Vega-Gálvez, \& Zura-Bravo, 2016). Estos antecedentes han incentivado el desarrollo de estudios que contemplen la domesticación de la especie y formulación de patentes que protejan variedades específicas con compuestos funcionales. La medicina tradicional ha atribuido a las partes aéreas de la planta, algunas propiedades astringentes, estimulantes y aromáticas; además, de diversas propiedades terapéuticas frente a afecciones de la piel y para el tratamiento de dolores del tracto urinario. Por ejemplo, la infusión de sus hojas es empleada para suavizar el cutis reseco y aumentar la elasticidad de la piel. En la actualidad se han desarrollado diferentes productos con formulaciones de extractos en hojas de esta especie nativa (Arancibia-Radich et al., 2016; Delporte et al., 2007; Goity et al., 2013). Otro aspecto de las hojas de Ugni molinae es su efecto inhibidor, por ejemplo se han reportado estudios de inhibición de la agregación de $\beta$ amiloide de extractos de hojas, esta molécula es capaz de agregarse en estructuras neurotóxicas que han sido implicadas como factores etiológicos potenciales de la enfermedad de Alzheimer (Jara-Moreno, Riveros, Barriga, Kogan, \& Delporte, 2020; Ma et al., 2019) y su actividad antiinflamatoria debido a los triterpenoides y el contenido fenólico total (Arancibia-Radich et al., 2016).

\subsection{Actividad antiinflamatoria}

Los extractos de hojas de murta han sido ampliamente utilizados por los pueblos originarios del sur de Chile (mapuche, puelche y pehuenche) para curar heridas y mejorar la piel deteriorada, por tener presente en sus bayas y hojas flavonoides, antocianinas y tocoferoles (López et al., 2017), los que están asociados a una alta actividad antioxidante (Hauser, Peñaloza, Rodriguez, Guarda, \& Galotto, 2014; Peña-Cerda et al., 2017); estos les confiere propiedades anti-inflamatorias y contienen diferentes tipos de compuestos fenólicos, tales como ácido cafeico, quercetina, rutina, ácido gálico, kaempferol (Arancibia-Radich et al., 2016; Chacón-Fuentes et al., 2015; Delporte et al., 2007). Otro ejemplo es la presencia de la indometacina, utilizada como antiinflamatorio, que se presenta en las hojas y frutos de la murta; dependiendo de las condiciones agronómicas (Peña-Cerda et al., 2017). En este sentido las hojas de murta han sido utilizadas en la medicina tradicional chilena como infusión por su actividad anti-inflamatoria (Arancibia-Radich et al., 2016; D'Urso et al., 2019; Schmeda-Hirschmann et al., 2019).

El contenido de estos compuestos en las hojas de murta son variables y dependen en gran medida en las concentraciones de sus ácidos triterpenoides, en los contenidos totales de fenoles, (Arancibia-Radich et al., 2016; Delporte et al., 2007), y particularmente en el contenido de las antocianinas (Ulloa-Inostroza et al., 2017). Algunas investigaciones relacionan las hojas de murta con la presencia de triterpenos de estructura 
pentacíclica, los cuales les dan una significativa actividad analgésica y antiinflamatoria tópica (Chakraborty et al., 2019), y los flavonoides y taninos con la actividad antioxidante (Peña-Cerda et al., 2017). Otro componente asociado a la actividad antiinflamatoria y con propiedades cicatrizantes es el ácido asiático, el cual es conocido como uno de los componentes activos inhibidor de la fase inflamatoria de las cicatrices y queloides hipertróficas. Por su composición fenólica y capacidad antioxidante los extractos de hojas de murta se utilizan también como analgésicos y antiinflamatorios por su efecto antinociceptivo y podrían ser la razón de su amplio uso en la medicina popular para tratar diferentes tipos de dolor (Delporte et al., 2007; Peña-Cerda et al., 2017). En la Tabla 2 se reportaron los componentes asociados a la actividad anti- inflamatoria en la murta.

\subsection{Actividad antimicrobiana}

En la actualidad existe gran preocupación por la inocuidad microbiológica de los alimentos y su vida útil (Lin \& Tsao, 2019), el diseño de nuevos envases que conserven el producto durante un periodo de tiempo mayor ha llamado mucho la atención como tecnología innovadora para la conservación de los alimentos (Ahmad, Dar, Abas, \& Ahmad, 2018; López et al., 2016; Lopez et al., 2016; Pacheco et al., 2019), por ello los agentes antimicrobianos que tienen como principal objetivo inhibir a los microorganismos presentes en los alimentos, tienen actualmente una gran importancia por reducir los procesos naturales de deterioro, previniendo y controlando el crecimiento de microorganismos patógenos y de otros causantes de deterioro (ArancibiaRadich et al., 2016; Shene et al., 2009; Tajkarimi, Ibrahim, \& Cliver, 2010). Se sabe que los aceites derivados de plantas aromáticas y algunas especies silvestres tienen un efecto antimicrobiano de acción sobre las levaduras, mohos y bacterias (Adam, Dobiáš, Pavlíková, \& Ventura, 2009; Tajkarimi et al., 2010).

Las nuevas aplicaciones de la fruta y las hojas de murta, de origen natural o como extracto en alimentos con propiedades funcionales (Ahmad et al., 2018) pueden ser interesantes gracias a su actividad antimicrobianas (Lin \& Tsao, 2019; López de Dicastillo et al., 2017). Por ejemplo, los extractos de hojas de murta hidrodestiladas en estado vegetativo y de floración, son eficaces en la eliminación de bacterias e inhibe el crecimiento de hongos o parásitos por su actividad antimicrobiana (Hauser et al., 2014; Tajkarimi et al., 2010). Hauser et al. (2014) determinaron actividad antimicrobiana y antioxidante en diferentes extractos de hojas de murta $(1,5 \mathrm{~g}$ de hojas de murta obtenido con $20 \mathrm{ml}$ de etanol al 50\%), el cual disminuyo el crecimiento de Listeria innocua (ATCC 33090) inhibiendo 4,9 y 4,8 ciclos log después de 2 y 24 h de contacto respectivamente. En la Tabla 3 se reportaron componentes asociados a la actividad antimicrobianas en la murta.

\subsection{Capacidad antioxidante}

Los antioxidantes juegan un papel muy importante en el sistema de defensa del cuerpo contra especies reactivas de oxígeno (ROS), los antioxidantes son un inhibidor del proceso de oxidación, por lo tanto, tienen un papel fisiológico diverso en el cuerpo. Los componentes antioxidantes de las plantas actúan como carroñeros de radicales y ayudan a convertir los radicales en especies menos reactivas, en la fruta y vegetales se encuentran las fuentes dietéticas que aportan antioxidantes que sirven para la neutralización de radicales libres (Mandal, Yadav, Yadav, \& Kumar, 2009), estos componentes antioxidantes incluyen: carotenos, vitamina C, fenoles, flavonoides, glutatión dietético y metabolitos endógenos.

Tabla 2

Componentes asociados a la actividad anti-inflamatoria en la murta (Ugni molinae)

\begin{tabular}{|c|c|c|c|c|c|}
\hline \multirow{2}{*}{ Componentes } & \multirow{2}{*}{ Actividad o función } & \multicolumn{3}{|c|}{ Ubicación } & \multirow[t]{2}{*}{ Referencias } \\
\hline & & Hojas & Tallo & Fruto & \\
\hline $\begin{array}{l}\text { Diclorometano acetato de etilo y } \\
\text { metanol }\end{array}$ & Efecto antinociceptivo & $\begin{array}{c}\text { Extractos de } \\
\text { hojas } \\
\text { Seco }\end{array}$ & & & (Delporte et al., 2007) \\
\hline $\begin{array}{l}\text { Contenido fenólico y } \\
\text { triterpenoides }\end{array}$ & Anti-inflamatorio & $\begin{array}{l}\text { Hojas frescas y } \\
\text { secas }\end{array}$ & & & $\begin{array}{l}\text { (Arancibia-Radich et } \\
\text { al., 2016) }\end{array}$ \\
\hline $\begin{array}{l}\text { Triterpenoides } \\
\text { (ácido madecásico, ácido } \\
\text { alfitólico, ácido maslínico) }\end{array}$ & Anti-inflamatorio & $\begin{array}{l}\text { Extractos de } \\
\text { hoja en etanol }\end{array}$ & & & (Goity et al., 2013) \\
\hline Polifenoles & $\begin{array}{c}\text { Antiemolítico, } \\
\text { suplementos } \\
\text { nutricionales o } \\
\text { medicinales, } \\
\text { Antioxidante }\end{array}$ & Extracto crudo & & & (Rubilar et al., 2011) \\
\hline Compuestos fenólicos & $\begin{array}{l}\text { Antiinflamatorios y } \\
\text { analgésicos }\end{array}$ & Extracto seco & & Extracto & $\begin{array}{l}\text { (Cabrera-Barjas et al., } \\
\text { 2020) }\end{array}$ \\
\hline
\end{tabular}


Tabla 3

Componentes asociados a la actividad antimicrobianas en la murta (Ugni molinae)

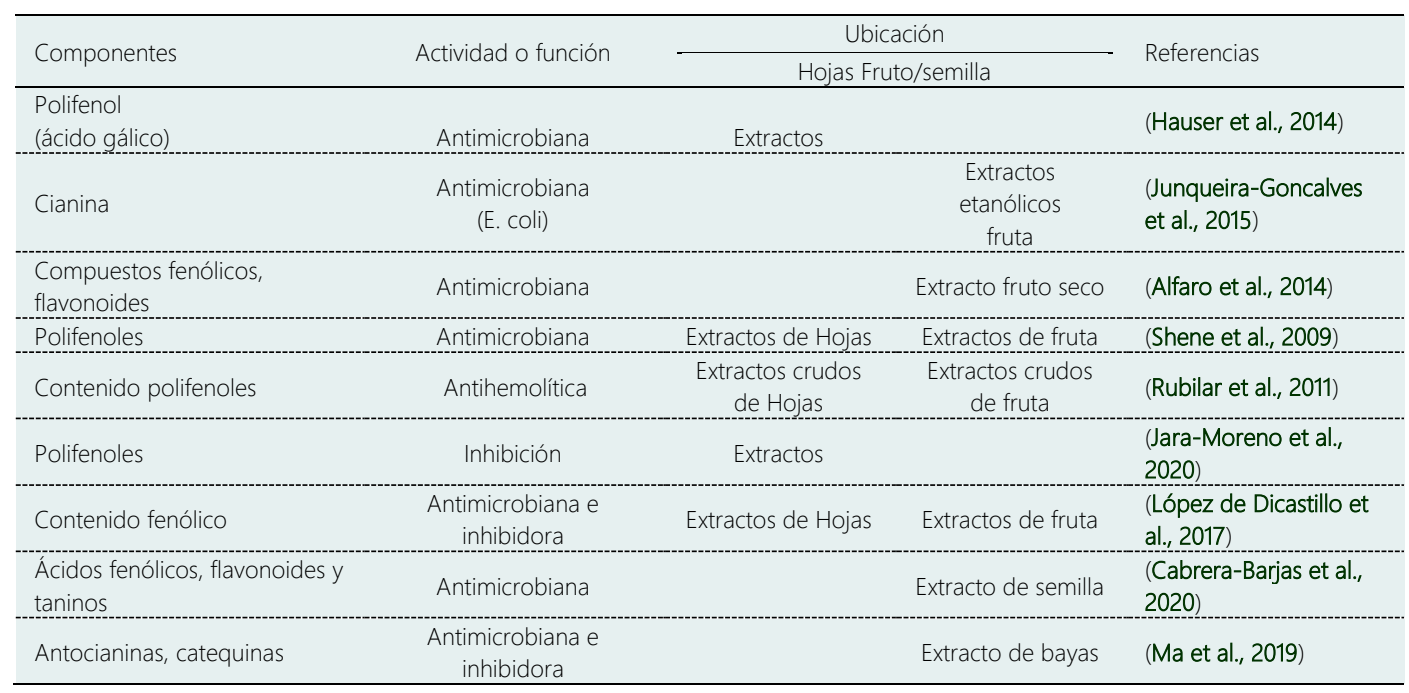

Los extractos de murta contienen muchos componentes con capacidad antioxidante en comparación con el maqui chileno y el calafate (Augusto-Obara et al., 2017; Junqueira-Goncalves et al., 2015; López et al., 2017). Los extractos de hojas de murta han mostrado contener un alto contenido de taninos y flavonoides (Doll, Rodríguez, Soto, \& Razmilic, 2012); debido a esto, cada vez más se está incorporando al desarrollo de alimentos, productos naturales con propiedades antioxidantes, cuyos extractos ricos en polifenoles pueden beneficiarnos con su consumo (Pacheco et al., 2019). Los extractos de hojas de murta pueden aportar propiedades reológicas dependiendo de su composición y pueden ser utilizadas para obtener recubrimientos o películas biodegradables para aplicaciones alimentarias, farmacéuticas 0 cosméticas y la vida útil (Chakraborty et al., 2019; PeñaCerda et al., 2017; Sabando et al., 2020; Silva-Weiss, Bifani, Ihl, Sobral, \& Gómez-Guillén, 2014). Otras investigaciones han mostrado la actividad antioxidante en infusiones de murta al 1\%, junto con el incremento en la actividad antioxidante plasmática asociada a su ingesta (ArancibiaAvila et al., 2011; Arancibia-Radich et al., 2016; Fredes, Parada, Salinas, \& Robert, 2020). Otros resultados muestran que el consumo la murta reduce el riesgo de trastornos de enfermedades cardiovasculares como la hipertensión, debido a la capacidad antioxidantes y su asociación con la prevención de trastornos fisiopatológicos en seres humanos, tales como inflamación y diabetes, debido a su contenido de fenoles (Ah-Hen et al., 2018; Fredes et al., 2020; Jofre et al., 2016; Kamiloglu et al., 2016; Ulloa-Inostroza et al., 2017). Fenoles en extractos secos de hojas de murta han sido encontrados en concentraciones de 30,9 - 53,55 (mg QE/g DE) (Peña-Cerda et al., 2017), también han sido reportados concentraciones de polifenoles en extracto crudo de hojas de murta entre 32,5 $\pm 3,1 \mathrm{mg} \mathrm{GAE} / \mathrm{g} \mathrm{dm}$ ) y en tallos de hojas entre 15,8 \pm 0,2 mg GAE / g dm (Rubilar et al., 2011).

En general el primer factor condicionante del perfil polifenólico en la murta, son las condiciones climáticas extremas que pueden aumentar el contenido de polifenoles en las hojas (Pacheco et al., 2019), siendo sus extractos, más concentrados y con mayores principios bioactivos (Alfaro et al., 2014). En la Tabla 4 se reportan los componentes asociados a la capacidad antioxidantes en la murta.

\section{Otros usos de la murta}

De acuerdo a la Organización Mundial de la Salud (OMS), las plantas medicinales son todas aquellas que en uno o más órganos contienen sustancias útiles con fines terapéuticos o que sirven como precursores para la hemisíntesis química. Las hojas o plantas han sido un acompañante constante en la historia del ser humano como alimento, medicina, ornato y veneno (Barreau, Ibarra, Wyndham, Rojas, \& Kozak, 2016; Cortés \& Calderón, 2019). La gran importancia medicinal que se le ha atribuido a la murta, ha motivado a la ciencia a buscar respuestas y es así como investigaciones han identificado altas propiedades medicinales y de uso cosmético de la murta por los altos contenidos de polifenólicos, terpenos y taninos (Doll et al., 2012), estos elementos hacen que la murta sea muy atractiva para la industria dermatológica por sus potentes cualidades regeneradoras de tejidos o cicatrizantes, por sus efectos correctivos y neutralizantes del estrés oxidativo. La murta presenta un alto contenido de pectina $(0,32-1,14 \%)$, lo cual es un valioso ingrediente funcional ampliamente empleado como agente gelificante, emulsionante y estabilizante; principalmente es utilizado para hacer infusiones por nativos para curar la diarrea y la disentería. También se hacen mermeladas, jaleas, jugos de frutas, té, concentrados de bebidas de frutas, postres, debido a su poder gelificante (Ah-Hen et al., 2018; Arancibia-Avila et al., 2011; J. López et al., 2018; Ulloa-Inostroza et al., 2017). Otros usos de los extractos de hojas de murta, es su utilización en la industria cosmética y farmacéutica, ávidas actualmente de antioxidantes de origen natural; y que requieren de materias primas homogéneas de alta calidad (Doll et al., 2012). También es apreciada por sus propiedades 
descongestionante, regenerativas y de antienvejecimiento. Debido a que los extractos de frutos de murta son una fuente natural de antioxidantes para la protección de los alimentos lipídicos (Augusto-Obara et al., 2017), se utilizan en la elaboración de películas de envasado activo por su alto contenido de antioxidante y por su actividad antimicrobiana (Lin \& Tsao, 2019), para prolongar la vida útil de los alimentos envasados (Ahmad et al., 2018; Hauser et al., 2016). Los extractos de murta también han sido utilizados en el control de insectos herbívoros (Chacón-Fuentes et al., 2015). El cuero de frutas es un producto de baja humedad y estable a temperatura ambiente y pueden ser una novedosa alternativa de producción por sus propiedades antioxidantes en el uso de hidrocoloides como la gelatina (Gomez-Perez, Navarrete, Moraga, Rodriguez, \& VegaGalvez, 2020).

\section{Aspectos económicos de la murta}

La murta comercializada en Chile proviene mayoritariamente de la recolección silvestre, encontrándose los mayores volúmenes de comercialización en las distintas ferias de chile: Osorno, Valdivia, Temuco, Puerto Montt y en Ensenada. El período de cosecha y venta se realiza usualmente de febrero a mayo, siendo muy variable dependiendo de la frecuencia y persistencia de las lluvias otoñales que oscilan entre 1000 a 3000 mm (Valdebenito, 2009). La recolección en promedio fluctúa entre 10 y 20 kg por persona al día. El producto una vez recolectado, es transportado hasta las ciudades y vendido a los intermediarios y a los consumidores finales. La otra línea de comercialización es aquella que se efectúa a través de una empresa exportadora. En este caso, es la misma empresa la que compra a los recolectores, quienes recolectan la murta desde sus propios predios, desde predios de vecinos y también desde predios propios de la empresa exportadora mediante un sistema de trato por kilogramo recolectado (Aguila \& Nahuelhual, 2008; Seguel \& Torralbo, 2004; Valdebenito, 2009). En la Figura 2 se muestra el esquema del proceso de comercialización interno y externo en la zona sur de Chile. Respecto al precio de la murta y sus productos procesados, la planta procesadora de alimentos de la Universidad de Los Lagos, Osorno, Chile, estableció valores de mercado para el año 2021 de los siguientes productos: El precio del kilo de murta fresca a USD 2,82, el kilo de pulpa congelada a USD 7,05, mermelada de murta (500 g) a USD 4,23, y el jugo de murta $(250 \mathrm{ml}$ ) a USD 2,18. Se estima que los volúmenes totales de murta tranzados en el mercado, son a los menos 16 toneladas por temporada, a través de las diferentes vías de comercialización y 800 kg por recolector en cada temporada.

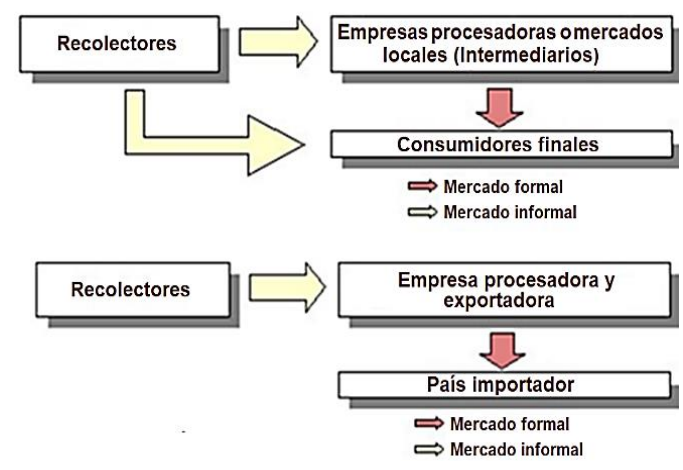

Figura 2. Esquema del proceso de comercialización interno y externo en la zona sur de Chile. Adaptado desde Valdebenito et al. (2011).

\section{Tabla 4}

Componentes asociados a la capacidad antioxidante en la murta (Ugni molinae)

\begin{tabular}{|c|c|c|c|c|}
\hline \multirow{2}{*}{ Componentes } & \multirow{2}{*}{ Actividad o función } & \multicolumn{2}{|c|}{ Ubicación } & \multirow{2}{*}{ Referencias } \\
\hline & & Hojas & Fruto & \\
\hline $\begin{array}{l}\text { Polifenoles, antocianina y } \\
\text { carotenoides }\end{array}$ & Fenólicos bioactivos & & $\begin{array}{l}\text { Extracto de } \\
\text { jugo }\end{array}$ & ( Ah-Hen et al., 2018) \\
\hline Contenido fenólico total & Antioxidante & & $\begin{array}{l}\text { Extractos } \\
\text { hidroalcohólicos } \\
\text { de frutas }\end{array}$ & $\begin{array}{l}\text { (Augusto-Obara et } \\
\text { al., 2017) }\end{array}$ \\
\hline Contenido fenólico & Antioxidante & $\begin{array}{c}\text { Extractos de hojas } \\
\text { acuosas }\end{array}$ & & (Avello et al., 2013) \\
\hline $\begin{array}{l}\text { Contenido fenólico } \\
\text { (ácido gálico, catequina, quercetina- } \\
\text { 3- } \beta \text {-D-glucósido, miricetina, } \\
\text { quercetina y kempferol) }\end{array}$ & Vasodilatador & & Fruta nativa & (Jofre et al., 2016) \\
\hline Tanino y flavonoide & Antioxidante & Hojas & & (Doll et al., 2012) \\
\hline $\begin{array}{l}\text { Contenido total de fenóles y } \\
\text { flavonoides }\end{array}$ & $\begin{array}{c}\text { Antioxidantes y } \\
\text { compuestos bioactivos }\end{array}$ & & Fruto & $\begin{array}{l}\text { (Rodriguez et al., } \\
\text { 2014) (Ah-Hen et al., } \\
\text { 2018) }\end{array}$ \\
\hline Flavonoides & Compuestos bioactivos & & Fruto seco & (López et al., 2017) \\
\hline Antocianinas & $\begin{array}{l}\text { Composición } \\
\text { nutricional y } \\
\text { fitoquímica }\end{array}$ & Hojas & Fruto & (López et al., 2018) \\
\hline $\begin{array}{l}\text { Quercetina, } \\
\text { epicatequina, y ácido gálico }\end{array}$ & Antioxidante & & $\begin{array}{l}\text { Extractos } \\
\text { hidroalcohólicos }\end{array}$ & $\begin{array}{l}\text { (Riquelme et al., } \\
\text { 2014) }\end{array}$ \\
\hline $\begin{array}{l}\text { Pectina, ácido ferúlico, ácido } \\
\text { galacturónico }\end{array}$ & $\begin{array}{l}\text { Composición de los } \\
\text { monosacáridos }\end{array}$ & & Extractos & $\begin{array}{l}\text { (Chakraborty et al., } \\
\text { 2019) }\end{array}$ \\
\hline Compuestos fenólico y flavonoide & Antioxidante & $\begin{array}{l}\text { Extractos } \\
\text { etanólicos de } \\
\text { hojas }\end{array}$ & & $\begin{array}{l}\text { (Peña-Cerda et al., } \\
\text { 2017) }\end{array}$ \\
\hline
\end{tabular}


También, se han reportado estudios de factibilidad económica en cultivo de murta, en dos escenarios; el primero considera las recomendaciones técnicas del Instituto Nacional de Investigación Agraria (INIA) en Chile, tal como se presentan en el protocolo del cultivo y considera tanto la comercialización nacional (mercados locales y agroindustrias) como internacional (exportación vía intermediarios). Bajo este escenario, se asume que un $20 \%$ de la producción se destina al mercado local, un 30\% al mercado nacional (agroindustrias) y un 50\% a exportación. El segundo escenario (escenario alternativo), que se adapta mayormente a las condiciones existentes en el área de estudio, considera la producción propia de plantas, la existencia de una bonificación al riego y una comercialización restringida al mercado local y regional, destinando un $70 \%$ del volumen producido a agroindustrias y un 30\% al mercado local (Valdivia), Región de Los Rios, Chile. El valor actual de los beneficios netos (VABN) del cultivo bajo este escenario original, a un horizonte de tiempo de 10 años, fue de USD 22148,16 ha-1; con una tasa interna de retorno (TIR) de 33,9\%. En el escenario alternativo, el VABN disminuyó a USD 6884,60 $\mathrm{ha}^{-1}$ con una TIR de $25 \%$, indicando la alta rentabilidad del cultivo para ambos escenarios (Aguila \& Nahuelhual, 2008; Seguel \& Torralbo, 2004; Torralbo, 2011). En la Figura 3 se muestra el volumen exportado (expresado en kg) en Chile, entre el 2010 y 2017. Se puede observar que existe una gran fluctuación en los volúmenes de las exportaciones; esto puede ser debido a que todavía la producción de murta es informal y depende mucho de los recolectores artesanales de fruta, sin embargo, este producto existe en muchas presentaciones y los indicadores apuntan hacia potenciales mercados como es el caso de Alemania, Francia, Italia (ver Figura 4); pero antes se debe asegurar la calidad y un monto estable de oferta para lograr realizar un negocio serio y sostenible (Agricultura, 2017).

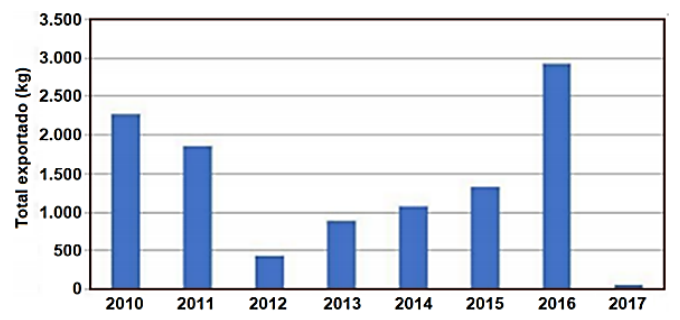

Figura 3. Volumen exportado en Chile, entre 2010 a 2017. Ministerio de Agricultura (2017)

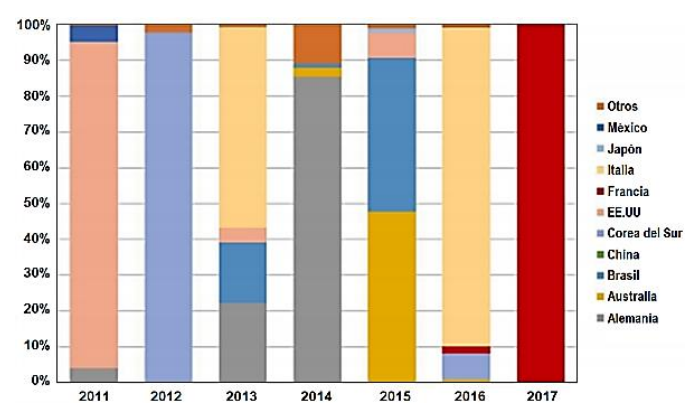

Figura 4. Distribución de exportaciones de murta en Chile del 2011 a 2017, por país de destino. Ministerio de Agricultura (2017).

\section{Retos actuales y futuros}

La murta es un berries silvestre con grandes oportunidades, de modo de capturar y potenciar sus atributos funcionales para el desarrollo de productos e ingredientes con beneficios para la salud. Sin embargo, hay que poner atención a algunos retos actuales y futuros que se presentan, de modo de no opacar su potencial natural. Un desafío común a todos es el posicionamiento en el mercado internacional, lo que implica un tremendo esfuerzo y lo que requiere de muchos años para lograr entrar en la retina de los consumidores. La tendencia actual es consumir alimentos funcionales con propiedades nutricionales especiales, observándose que aquellos consumidores que antes dependían en gran medida de los suplementos alimenticios, y que hoy están cambiando al consumo de alimentos fortificados y funcionales en su reemplazo. Por ejemplo, por consumir más nutrientes como: vitaminas, minerales, hierbas, productos botánicos e incluir más pescado, aceite y omega-3 en sus dietas (Fuentes et al., 2019; López et al., 2018; Santander et al., 2017; SchmedaHirschmann et al., 2019; Zura-Bravo et al., 2019; ZuraBravo et al., 2018)

Una demanda más permanente de este berries podría establecerse mediante la incorporación de un valor agregado, que sea atractivo y conserve las características de calidad de las materias primas. En este sentido, se puede mencionar la gran cantidad de productos en esta línea que se comercializan principalmente en supermercados y tiendas especializadas de Estados Unidos y Europa, como, por ejemplo, bebidas energizantes, bebidas para deportistas, bebidas antioxidantes libres de calorías, bebidas herbales con mezcla de berries y hierbas, bebidas para conciliar el sueño, mezclas de jugos concentrados, mermeladas orgánicas, aderezos bajos en calorías y té orgánicos, entre otros. Barras de proteínas donde se mezclan berries con chocolate o simplemente berries con proteínas de suero de leche también son otros de los productos encontrados en el mercado internacional. A su vez, el té verde es desde hace unos años el té más consumido en Estados Unidos, por lo que infusiones sobre la base de berries estudiados mezclados con té verde, sería una apuesta interesante para complementar aún más sus niveles basales de antioxidante (Chile \& Adimark, 2014). Fomentar, desde el Estado, los modelos de gestión asociativos, de pequeña escala, territoriales, con base en los recolectores y personas que poseen los conocimientos tradicionales. Los bosques y los árboles brindan contribuciones decisivas tanto a las personas como al planeta al fortalecer los medios de vida, suministrar aire y agua limpia, alimentos, conservar la biodiversidad y responder al cambio climático (Engels \& Thormann, 2020; Miina, Pukkala, \& Kurttila, 2016; Orchard, Munoz-Poblete, Huircan, Galeas, \& Rozas, 2019).

Si bien se ha avanzado en el desarrollo agronómico y cultivo comercial de la murta, hoy Chile se enfrenta al desafío de aumentar sus áreas de producción y abordar un posicionamiento internacional a través del marketing, de modo de hacer frente a la competencia de países como Australia y Nueva Zelanda, quienes ya están incorporando la murta como producto premium en el mercado gourmet (Barrios \& Cruzat, 2009; Chile \& Adimark, 2014). 


\section{Conclusiones}

El presente trabajo de revisión realizó una recopilación de información actualizada de investigaciones referente a la murta, un fruto alimenticio con propiedades funcionales y/o con compuestos bioactivos como por ejemplo las antocianinas, polifenoles y carotenos, los que les confieren componentes asociados a la actividad antiinflamatoria, actividad antimicrobiana, capacidad antioxidante, composición nutricional. Según lo reportado por diversos investigadores se concluye que la murta es una buena fuente de alimentación con propiedades funcionales y benéficas para la salud humana. Investigaciones futuras se deben realizar para confirmar si es estos componentes mantienen su actividad biológica durante almacenamiento prolongado, procesamiento y por la ingesta del fruto. También se deben realizar investigaciones futuras de sus semillas por su alto grado de ácidos grasos insaturados como consecuencia de su elevado contenido de ácido linoleico, lo que mejora su potencial como aceite comestible. Además, se recomienda realizar investigaciones de algunos tratamientos térmicos que se utilizan en el procesamiento de la murta y ver si se pierden algunos componentes benéficos. Por último, se recomienda realizar estudios económicos actualizados de murta en cuanto a la producción, comercialización y exportaciones.

\section{Agradecimientos}

Programa de Magíster Latinoamericano en Administración de Empresas y Negocios, D. U. No 3165, Dirección de Posgrado, Vicerrectoría de Investigación y Postgrado, Universidad de Los Lagos, Osorno, Chile.

\section{ORCID}

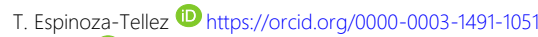

J. Bastías (D) https://orcid.org/0000-0003-3387-6917

R. Quevedo-León (DD https://orcid.org/0000-0001-8132-838X

E. Valencia-Aguilar (D) https://orcid.org/0000-0001-5748-9415

O. Díaz-Carrasco $\mathbb{D}_{\text {https://orcid.org/0000-0002-0937-9825 }}$

M. A. Solano (D) https://orcid.org/0000-0002-8221-7443

F. Mesa-Mesina (D) https://orcid.org/0000-0002-7695-8616

\section{Referencias bibliográficas}

Adam, M., Dobiáš, P., Pavlíková, P., \& Ventura, K. (2009). Comparison of solidphase and single-drop microextractions for headspace analysis of herbal essential oils. Cent. Eur. J. Chem., 7(3), 303-311.

Agricultura, M. D. (2017). Preparación de expedientes técnicos para la presentación y solicitud de autorización de alimentos nuevos o tradicionales de terceros países para exportar a la unión europea. Odepa, $1-126$

Aguila, C., \& Nahuelhual, L. (2008). cultivo de murtilla (ugni molinae turcz.) como alternativa de diversificación productiva para la agricultura familiar campesina de la cordillera de la costa. Agro sur, 36(3), 158-167.

Ah-Hen, K., Lemus-Mondaca, R., Mathias-Rettig, K., Vega-Galvez, A., \& Lopez, J. (2014). Moisture Sorption Isotherms, Isosteric Heat of Sorption and Glass Transition Temperature of Murtilla (Ugni molinae T.) Berry. International Journal of Food Engineering, 10(4), 583-594.

Ah-Hen, K., Mathias-Rettig, K., Gómez-Pérez, L., Riquelme-Asenjo, G., LemusMondaca, R., \& Muñoz-Fariña, O. (2018). Bioaccessibility of bioactive compounds and antioxidant activity in murta (Ugni molinae T.) berries juices. Food Measure, 12, 602-615.

Ahmad, S., Dar, B., Abas, A., \& Ahmad, M. (2018). Efecto de los extractos de plantas sobre las propiedades tecno-funcionales de las películas de envasado biodegradables. Trends in Food Science \& Technology, 80, 141154.

Alarcón, D., López-Sepúlveda, P., Fuentes, G., Montoya, H., Peñailillo, P., \& Carrasco, P. (2019). Parches invadidos por Ugni molinae en isla Robinson Crusoe: ¿Hay plantas nativas y endémicas capaces de vivir en ellos? Gayana Botanica, 76(1), 126-131.
Alfaro, S., Mutis, A., Quiroz, A., Seguel, I., \& Scheuermann, E. (2014). Effects of Drying Techniques on Murtilla Fruit Polyphenols and Antioxidant Activity Journal of Food Research, 3(5), 73-82.

Arancibia-Avila, P., Toledo, F., Werner, E., Suhaj, M., Leontowicz, $H_{\text {., }}$ et al. (2011). Partial characterization of a new kind of Chilean Murtilla-like berries. Food Research International, 44, 2054-2062.

Arancibia-Radich, J., Pena-Cerda, M., Jara, D., Valenzuela-Bustamante, P. Goity, L., et al. (2016). Comparative study of anti-inflammatory activity and qualitative-quantitative composition of triterpenoids from ten genotypes of Ugni molinae. Boletin latinoamericano y del caribe de plantas medicinales $y$ aromaticas, $15(5), 274-287$

Atala, C., \& Moya-Urrutia, V. (2014). Phytoplasma infection alters morphoanatomical and physiological traits in Ugni molinae Turcz. Gayana Bot., $77(1), 24-29$.

Augusto-Obara, T., Pirceb, F., Scheuermannb, E., Spoto, M., \& Vieira, T. (2017). Antioxidant activity and sensory analysis of murtilla (Ugni molinae Turcz.) fruit extracts in an oil model system. Grasas y aceites, 68(1), 1-9.

Avello, M., Pastene, E., Bustos, E., Bittner, M., \& Becerra, J. (2013). Variation in phenolic compounds of Ugni molinae populations and their potential use as antioxidant supplement Rev. Bras. Farmacogn. Braz. J. Pharmacogn., 23(1), 44-50

Avello, M., Pastene, E., \& Eillen, T. (2020). Identification of water-soluble compounds contained in aqueous extracts and fractions obtained from leaves of ugni molinae to determine their effect on the viability of human gastric cancer cells. Journal of the Chilean Chemical Society, 65(2), 48494852 .

Barreau, A., Ibarra, J., Wyndham, F., Rojas, A., \& Kozak, R. (2016). How Can We Teach Our Children if We Cannot Access the Forest? Generational Change in Mapuche Knowledge of Wild Edible Plants in Andean Temperate Ecosystems of Chile. Journal of Ethnobiology, 36(2), 412-443.

Barrios, E., \& Cruzat, R. (2009). Libro de valorizacion: resultados y lecciones en productos agroindustriales ricos en antioxidantes, a base de berries nativos. FIA Chile, 51.

Cabrera-Barjas, G., Quezada, A., Bernardo, Y., Moncada, M., Zúñiga, E., et al. (2020). Chemical composition and antibacterial activity of red murta (Ugni molinae Turcz.) seeds: an undervalued Chilean resource. Journal of Food Measurement and Characterization, 1-13.

Chacón-Fuentes, M., Parra, L., Rodriguez-Saona, C., Seguel, I., Ceballos, R., \& Quiroz, A. (2015). La domesticación en Murtilla ( Ugni molinae) redujo los niveles defensivos de flavonol pero aumentó la resistencia contra un insecto herbívoro nativo. Entomología ambiental, 44(3), 627-637.

Chakraborty, I., Sen, I., Mondal, S., Rout, D., Bhanja, S., et al. (2019). Bioactive polysaccharides from natural sources: A review on the antitumor and immunomodulating activities Biocatálisis y Biotecnología Agrícola, 22, 1-11.

Chile., F., \& Adimark. (2014). Chile saludable oportunidades y desafíos de innovación. Área Alimentos y Biotecnología de Fundación Chile, 3, 1-77.

Cortés, M., \& Calderón, F. (2019). Plantas medicinales chilenas: Desde el saber etnobotánico a los efectos terapéuticos y las reacciones adversas. Rev. méd. Chile vol.147 no.5 Santiago mayo 2019, 147(5), 673-676

D'Urso, G., Montoro, P., Lai, C., Piacente, S., \& Sarais, G. (2019). LC-ESI / LTQOrbitrap / MS metabolómica basada en el análisis de Myrtus communis parte de Cerdeña (Italia). Industrial Crops and Products, 128, 354-362.

Delporte, C., Backhouse, N., Inostroza, V., Aguirre, M., Peredo, N., et al. (2007) Analgesic activity of Ugni molinae (murtilla) in mice models of acute pain. Journal of Ethnopharmacology, 112(1), 162-165.

Doll, U., Rodríguez, I., Soto, C., \& Razmilic, I. (2012). Propagación de estacas y concentración de taninos y flavonoides en hojas de dos procedencias de Ugni molinae de la región del Maule (Chile) BOSQUE, 33(2), 203-209.

Engels, J., \& Thormann, I. (2020). Main Challenges and Actions Needed to Improve Conservation and Sustainable Use of Our Crop Wild Relatives. Plants-Basel, 9(8)

Fredes, C., Parada, A., Salinas, J., \& Robert, P. (2020). Phytochemicals and Traditional Use of Two Southernmost Chilean Berry Fruits: Murta (Ugni molinae Turcz) and Calafate (Berberis buxifolia Lam.). Foods, 9(1), 54.

Fuentes, L., Figueroa, C., Valdenegro, M., \& Vinet, R. (2019). Bayas patagónicas: potencial saludable y el camino para convertirse en alimentos funcionales: Review. Food, 8(8), 289

Goity, L., Queupil, M., Jara, D., Alegría, S., Peña, M., et al. (2013). An HPLC-UV and HPLC-ESI-MS based method for identification of antiinflammator triterpenoids from the extracts of Ugni molinae. Boletín Latinoamericano $y$ del Caribe de Plantas Medicinales y Aromáticas, 12(1), 108-116.

Gomez-Perez, L., Navarrete, C., Moraga, N., Rodriguez, A., \& Vega-Galvez, A. (2020). Evaluation of different hydrocolloids and drying temperatures in the drying kinetics, modeling, color, and texture profile of murta (Ugni molinae Turcz) berry leather. Journal of Food Process Engineering, 43(2), 13316.

Guenuleo, B., Torrego, S., Stefe, S., Neranzi, F., Moncunill, E., et al. (2020). Morpho-architectural and phenological characteristics of ugni molinae (myrtaceae): an andean patagonian shrub species with edible fruits. Boletin De La Sociedad Argentina De Botanica, 55(2), 215-230 
Hauser, C., Peñaloza, A., Guarda, A., Galotto, M., Bruna, J., \& Rodríguez, F. (2016). Development of an Active Packaging Film Based on a Methylcellulose Coating Containing Murta (Ugni molinae Turcz) Leaf Extract. Food Bioprocess Technol, 9, 298-307.

Hauser, C., Peñaloza, A., Rodriguez, F., Guarda, A., \& Galotto, M. (2014). Promising antimicrobial and antioxidant extracts of Murta leaves (Ugni molinae Turcz): Shelf-life extension and food safety. Food packaging and shelf life, 1(1), 77-85

Indap. (2018). Medicamentos hervarios tradicionales. Fucoa, Ministerio de Agricultura, 3, 1-227

Jaiswal, A., \& Abu-Ghannam, N. (2013). Kinetic studies for the preparation of probiotic cabbage juice: Impact on phytochemicals and bioactivity. Industrial Crops and Products, 50, 212-218.

Jara-Moreno, D., Riveros, A. L., Barriga, A., Kogan, M. J., \& Delporte, C. (2020). Inhibition of Beta-amyloid aggregation of Ugni molinae extracts. Current Pharmaceutical Design, 26(12), 1365-1376.

Jofre, I., Pezoa, C., Cuevas, M., Scheuermann, E., Freires, I. A., et al. (2016). Antioxidant and Vasodilator Activity of Ugni molinae Turcz. (Murtilla) and Its Modulatory Mechanism in Hypotensive Response. Oxidative Medicine and Cellular Longevity, 1-12.

Junqueira-Goncalves, M. P., Yanez, L., Morales, C., Navarro, M., Contreras, R. A., \& Zuniga, G. E. (2015). Isolation and Characterization of Phenolic Compounds and Anthocyanins from Murta (Ugni molinae Turcz.) Fruits. Assessment of Antioxidant and Antibacterial Activity. Molecules, 20(4), 5698-5713.

Kamiloglu, S., Toydemir, G., Boyacioglu, D., Beekwilder, J., Hall, R., \& Capanoglu, E. (2016). A Review on the Effect of Drying on Antioxidant Potential of Fruits and Vegetables. Crit Rev Food Sci Nutr, 56(1), 110-129.

Lemus-Mondaca, R., Ah-Hen, K., Vega-Gálvez, A., \& Zura-Bravo, L. (2016). Effect of high hydrostatic pressure on rheological and thermophysical properties of murtilla (Ugni molinae Turcz) berries. I Food Sci Technol, 53(6), 2725-2732

Lin, H., \& Tsao, R. (2019). Antimicrobianos de plantas - Conservación de alimentos y extensión de la vida útil. Comprehensive Biotechnology (Third Edition), 4, 703-714

López, C., Bustos, F., Guarda, A., \& Galotto, M. (2016). Cross-linked methyl cellulose films with murta fruit extract for antioxidant and antimicrobial active food packaging. Food Hydrocolloids, 60, 335-344

López de Dicastillo, C., Bustos, F., Valenzuela, X., López-Carballo, G., Vilariño, J., \& Galotto, M. (2017). Chilean berry Ugni molinae Turcz. fruit and leaves extracts with interesting antioxidant, antimicrobial and tyrosinase inhibitory properties. Food Research International, 102, 119-128.

Lopez, J., Ah-Hen, K., Vega-Gálvez, A., Morales, A., García-Segovia, P., \& Uribe, E. (2016). Efects of drying methods on quality attributes of murta (ugni molinae turcz) berries: bioactivity, nutritional aspects,texture profile, microstructure and functional properties. Food Process Engineering, 40(4), 1-11.

López, J., Vega-Gálvez, A., Rodríguez، A., Uribe, E.، \& Bilbao-Sainz, C. (2018). Murta (ugni molinae turcz.): a review on chemical composition, functional components and biological activities of leaves and fruits: Review. Chilean J. Agric. Anim. Sci., ex Agro-Ciencia, 34(1), 1-14

López, J., Vega-Gálvez, A., Bilbao-Sainz, C., Bor-Sen, C., Uribe, E., \& Quispe Fuentes, I. (2017). Influencia de la temperatura de secado al vacío en: composición fisicoquímica y propiedades antioxidantes de las bayas de murta. J Food Process Eng, 40(6), 1-9

Lorca, M. (2018). Chilean Guava-Myrtus ugni. Exotic Fruits Reference Guide, 129-139.

Ma, Y., Ding, S., Fei, Y., Liu, G., Jang, H., \& Fang, J. (2019). Antimicrobial activity of anthocyanins and catechins against foodborne pathogens Escherichia coli and Salmonella. Food Control, 106, 1-8

Mandal, S., Yadav, S., Yadav, S., \& Kumar, R. (2009). Antioxidants: A Review Journal of Chemical and Pharmaceutical Research, 1(1), 102-104.

Miina, J., Pukkala, T., \& Kurttila, M. (2016). Optimal multi-product management of stands producing timber and wild berries. European Journal of Forest Research, 735(4), 781-794.

Nowakowska, M., Ochmian, I., \& Mijowska, K. (2017). The influence of street conditions on sea buckthorn fruit quality and content of micro- and macronutrients in berries and in soil. Journal of Elementology, 22(1), 235 244.

Orchard, M., Munoz-Poblete, C., Huircan, J., Galeas, P., \& Rozas, H. (2019). Harvest Stage Recognition and Potential Fruit Damage Indicator for Berries Based on Hidden Markov Models and the Viterbi Algorithm. Sensors, 19(20).

Pacheco, N., Naal-Ek, M., Ayora-Talavera, T., Shirai, K., Román-Guerrero, A. et al. (2019). Efecto del quitosano bioquímico y el ácido gálico en las propiedades reológicas y fisicoquímicas de las películas comestibles ternarias. Macromolecules, 25, 149-158.

Parkar, S., Stevenson, D., \& Skinner, M. (2008). The potential influence of fruit polyphenols on colonic microflora and human gut health. Int J Food Microbiol, 124(3), 295-298

Peña-Cerda, M., Arancibia-Radich, J., Valenzuela-Bustamante, P., PérezArancibia, R., et al. (2017). Phenolic composition and antioxidant capacity of Ugni molinae Turcz. leaves of different genotypes. Food Chemistry, 215, 219-227.

Pereira, C., da Silva, E., de Souza, A., Vieira, M., Ribeiro, A., \& Cadore, S. (2018), Evaluation of the bioaccessibility of minerals from blackberries, raspberries, blueberries and strawberries. Journal of Food Composition and Analysis, 68, 73-78.

Petousi, I., Daskalakis, G., Fountoulakis, M., Lydakis, D., Fletcher, L., et al. (2019). Effects of treated wastewater irrigation on the establishment of young grapevines. Science of the Total Environment, 658, 485-492.

Pirce, F., Vieira, T., Augusto-Obara, T., Alencar, S., Romero, F., \& Scheuermann, E. (2020). Effects of convective drying assisted by ultrasound and osmotic solution on polyphenol, antioxidant and microstructure of murtilla (Ugni molinae Turcz) fruit. Journal of Food Science and TechnologyMysore, 58, 138-146.

Radrigán, R., Aqueveque, P., \& Ocampo, M. (2017). Capítulo 7 - Extracción y uso de ingredientes funcionales de plantas para el desarrollo de alimentos funcionales. Manual de Bioingeniería de Alimentos, 275-306.

Ramirez, J., Zambrano, R., Sepúlveda, B., Kennelly, E., \& Simirgiotis, M. (2015). Anthocyanins and antioxidant capacities of six Chilean berries by HPLCHR-ESI-ToF-MS. Food Chemistry, 176(9), 106-114.

Retamales, H., Scherson, R., \& Scharaschkin, T. (2014). Foliar micromorphology and anatomy of Ugni molinae Turcz. (Myrtaceae), with particular reference to schizogenous secretory cavities. Revista Chilena De Historia Natural, 87(27), 1-7.

Riquelme, T., Scheuermann, E., Alencar, S., Bismara, M., Costa de Camargo, A., \& Ferreira, M. (2014). Phenolic compounds and antioxidant activity of hydroalcoholic extracts of wild and cultivated murtilla (Ugni molinae Turcz.). Food Sci. Technol, 34(4), 667-673.

Rodriguez, K., Ah-Hen, K., Vega-Galvez, A., Lopez, J., Quispe-Fuentes, I., et al. (2014). Changes in bioactive compounds and antioxidant activity during convective drying of murta (Ugni molinae T.) berries. International Journal of Food Science and Technology, 49 (4), 990-1000.

Rodríguez, M., Carrillo, R., Chacón, M., Hormazábal, N., Tampe, J., \& Tighe, R. (2015). Enraizamiento in vitro y ex vitro de microtallos de Ugni molinae Turcz., una especie nativa de Chile. Gayana Bot., 72(1), 14-20.

Rodríguez, M., Hormazábal, N., Araneda, X., Tampe, J., Lobos, V., \& Castillo, C. (2016). Effects of gibberellic acid, benzylaminopurine and fluridone on the in vitro germination of Ugni molinae Turcz. (Myrtaceae). Gayana Bot., 73(1), 77-84

Rubilar, M., Jara, C., Poo, Y., Acevedo, F., Gutierrez, C., et al. (2011). Extracts of Maqui (Aristotelia chilensis) and Murta (Ugni molinae Turcz.): Sources of Antioxidant Compounds and r-Glucosidase/ r-Amylase Inhibitors. J. Agric. Food Chem, 59(5), 1630-1637.

Sabando, C., Ide, W., Rodriguez-Diaz, M., Cabrera-Barjas, G., Castano, J., et al. (2020). A Novel Hydrocolloid Film Based on Pectin, Starch and Gunnera tinctoria and Ugni molinae Plant Extracts for Wound Dressing Applications. Current Topics in Medicinal Chemistry, 20(4), 280-292

Santander, M., Osorio, O., \& Mejia- España, D. (2017). Evaluación de propiedades antioxidantes y fisicoquímicas de una bebida mixta durante almacenamiento refrigerado. revista de Ciencias Agrícolas, 34(1), 84-97.

Scheuermann, E., Ihl, M., Beraud, L., Quiroz, A., Salvo, S., et al. (2014). Effects of Packaging and Preservation Treatments on the Shelf Life of Murtilla Fruit (Ugni molinae Turcz) in Cold Storage. Packag. Technol. Sci. 2014; 27: , 27(3), 241-248.

Scheuermann, E., Seguel, I., Montenegro, M., Bustos, R., Hormazabal, E., \& Quiroz, A. (2008). Evolution of aroma compounds of murtilla fruits (Ugni molinae Turcz) during storage. J Sci Food Agric, 88(3), 485-492.

Schmeda-Hirschmann, G., Jiménez-Aspee, F., Theoduloz, C., \& Ladio, A. (2019). Bayas patagónicas como alimento y medicina nativos. Journal of Ethnopharmacology, 24, 1-27.

Seguel, I., \& Torralbo, L. (2004). Murtilla: El berrie nativo del sur de Chile. INIAFDI CORFO, 20-25.

Shene, C., Reyes, A., Villarroel, M., Sineiro, J., Pinelo, M., \& Rubilar, M. (2009). Plant location and extraction procedure strongly alter the antimicrobial activity of murta extracts. Eur Food Res Technol, 228, 467-475.

Silva-Weiss, A., Bifani, V., Ihl, M., Sobral, P., \& Gómez-Guillén, M. (2014). Polyphenol-rich extract from murta leaves on rheological properties of film-forming solutions based on different hydrocolloid blends. Journal of Food Engineering, 140, 28-38

Suwalsky, M., \& Avello, M. (2014). Antioxidant Capacity of Ugni molinae Fruit Extract on Human Erythrocytes: An In Vitro Study. J Membrane Biol, 247 703-712.

Taboada, E., Fisher, P., Jara, R., Zúñiga, E., Gidekel, M., et al. (2010). Isolation and characterisation of pectic substances from murta (Ugni molinae Turcz) fruits. Food Chemistry, 123(1), 669-678.

Tajkarimi, M., Ibrahim, S., \& Cliver, D. (2010). Review Antimicrobial herb and spice compounds in food. Food Control, 21, 1199-1218.

Torralbo, L. (2011). Aspectos económicos y de mercado de la murtilla. Proyecto FONDEF INIA, UACH, UFRO, 1-41.

Ulloa-Inostroza, E., Ulloa-Inostroza, E., Alberdi, M., Peña-Sanhueza, D. González-Villagra, J., et al. (2017). Native Chilean Fruits and the Effects of 
Their Functional Compounds on Human Health. Superfood and Functional Food - An Overview of Their Processing and Utilization, 1-33.

Valdebenito, G. (2009). Productos forestales no madereros en chile. Ciencia e Investigacion Forestal - Instituto Forestal Chile, 15 (1), 111 - 132

Valdebenito, G., Campos, J., Larraín, O., Aguilera, M., Kahler, C., et al. (2011). Información tecnológica de productos forestales no madereros del bosque nativo en chile Proyecto Conaf, 1-7.
Zura-Bravo, L., Rodríguez, A., Stucken, K., Castillo, L., Ah-Hen, K., et al. (2019). Propiedades nutricionales y organolépticas de las bayas de murta (Ugni molinae Turcz) impregnadas con Lactobacillus casei var. ramnoso y deshidratado por diferentes métodos. Química de Alimentos, 299, 1-7.

Zura-Bravo, L., Rodriguez, A., Stucken, K., \& Vega-Gálvez, A. (2018). Drying kinetics of probiotic-impregnated murta (Ugni molinae T.) berries. J Food Sci Technol, 56, 103-113. 